
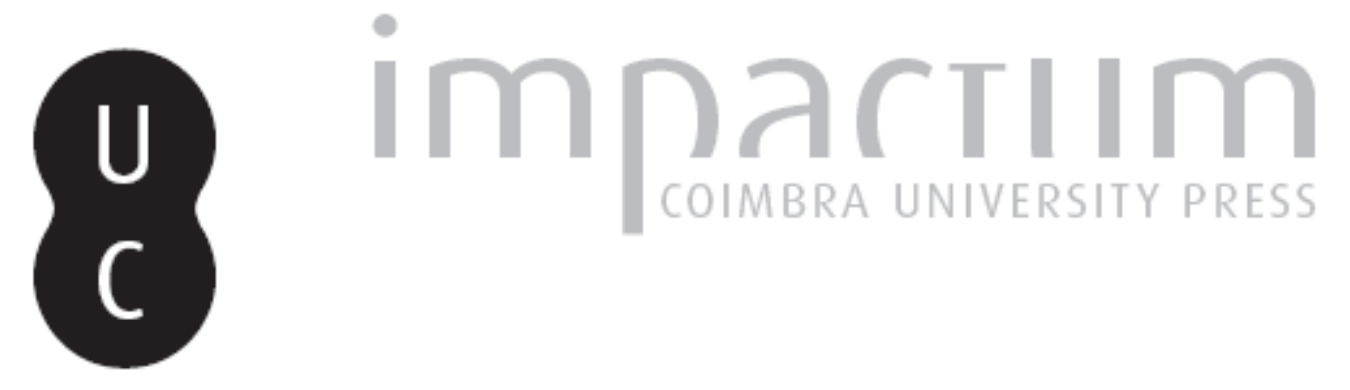

\title{
A Bibliotheca Alexandrina: um link para o passado e para o futuro
}

Autor(es): $\quad$ Sales, José das Candeias

Publicado por: Centro de História da Universidade de Lisboa

URL persistente:

URI:http://hdl.handle.net/10316.2/33013

DOI:

DOI:http://dx.doi.org/10.14195/0871-9527_22_12

Accessed : $\quad$ 26-Apr-2023 14:31:38

A navegação consulta e descarregamento dos títulos inseridos nas Bibliotecas Digitais UC Digitalis, UC Pombalina e UC Impactum, pressupõem a aceitação plena e sem reservas dos Termos e Condições de Uso destas Bibliotecas Digitais, disponíveis em https://digitalis.uc.pt/pt-pt/termos.

Conforme exposto nos referidos Termos e Condições de Uso, o descarregamento de títulos de acesso restrito requer uma licença válida de autorização devendo o utilizador aceder ao(s) documento(s) a partir de um endereço de IP da instituição detentora da supramencionada licença.

Ao utilizador é apenas permitido o descarregamento para uso pessoal, pelo que o emprego do(s) título(s) descarregado(s) para outro fim, designadamente comercial, carece de autorização do respetivo autor ou editor da obra.

Na medida em que todas as obras da UC Digitalis se encontram protegidas pelo Código do Direito de Autor e Direitos Conexos e demais legislação aplicável, toda a cópia, parcial ou total, deste documento, nos casos em que é legalmente admitida, deverá conter ou fazer-se acompanhar por este aviso.

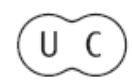



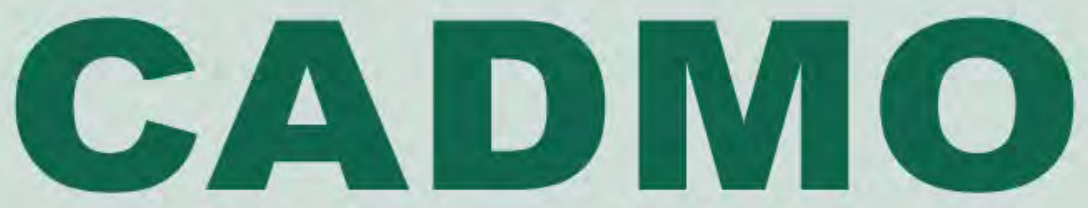

Revista de História Antiga

\author{
Centro de História \\ da Universidade de Lisboa
}

\title{
22
}

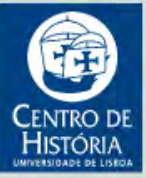

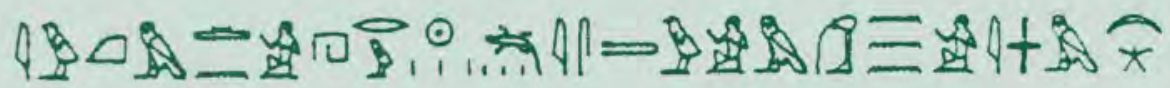

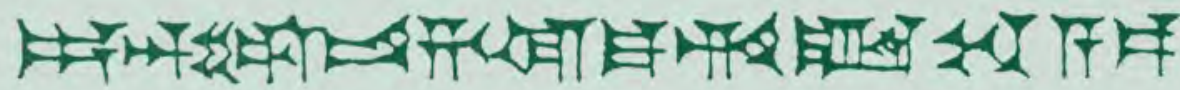
MHNIN AEI $\Delta \mathrm{E} \Theta \mathrm{EA} \Pi \mathrm{\Pi H} \Lambda \mathrm{HIA} \Delta \mathrm{E} \Omega$ 


\title{
A BIBLIOTHECA ALEXANDRINA: UM LINK PARA O PASSADO E PARA O FUTURO
}

\author{
JOSÉ DAS CANDEIAS SALES \\ Universidade Aberta, CHUL \\ jcsales@clix.pt
}

\begin{abstract}
"Some look at the world as it is and ask "why?", While we look the world as it could be, and ask "why not?"”

Bernard Shaw

«If you can imagine it, you can achieve it»
\end{abstract}

Goethe

\section{Resumo}

A Bibliotheca Alexandrina é muito mais do que um «simples» ícone arquitectónico do Egipto moderno. É o maior projecto cultural com que o Egipto entrou no século XXI. Em causa está a recuperação da ideia de um lugar de saber e de memória que se tornou mítico e se fixou no imaginário colectivo da própria humanidade.

Palavras-chave: Biblioteca; Alexandria; arquitectura; saber; cultura.

\section{Abstract}

The Bibliotheca Alexandrina is much more than a «simple» architectural icon of modern Egypt. Is the largest cultural project that Egypt entered the XXIst century. At issue is the recovery of the idea of a place of learning and memory that has become legendary, and settled in the collective imagination of Humanity itself.

Key-words: Library; Alexandria; architecture; knowledge; culture. 
Mesmo que nunca a tenham visitado in loco, mesmo que nunca tenham visto imagens concretas do seu edifício ou dos seus magníficos interiores, do seu soberbo enquadramento urbano-paisagístico junto da corniche (Avenida 26 de Julho) da baía da cidade, em «diálogo» com outros edifícios adjacentes e com inúmeras instituições a ela associadas (museus, galerias de arte, salas de exposição e de concertos, centro de conferências e de colóquios, planetário), não há praticamente ninguém que desconheça que na cidade egípcia de Alexandria existe actualmente uma nova biblioteca que, no essencial, pretende recuperar o lugar e a memória da antiga biblioteca, uma das glórias imemoriais da cidade.

16 de Outubro de 2002 assinalou a inauguração oficial pelo presidente egípcio Hosni Mubarak desta nova Bibliotheca Alexandrina (BA), um sonho muito antigo da cidade e do próprio Egipto, desde então um incontornável e renovado ponto de curiosidade e de atracção da segunda grande cidade egípcia, a norte do país. O início do século XXI via assim concretizar-se um projecto ambicioso que recuperava o espírito e os nobres valores da original biblioteca (instituir-se num centro de saber, de aprendizagem, de tolerância, de diálogo, de compreensão e de cooperação dos povos e das culturas), ao mesmo tempo que reanimava a carga simbólica que a posteridade construiu e consolidou para a biblioteca dos tempos antigos.

A antiga biblioteca de Alexandria (megale bibliotheke) foi reconhecidamente uma das mais ambiciosas e excitantes aventuras intelectuais da humanidade: «For the first time in history an attempt was made to collect universal knowledge, codify and organize it, and open up cultures to each other. This ecumenical spirit, with its commitment to tolerance, diversity, and openness to the other, was accompanied by a strong commitment to rationality, debate and the methods of scholarship (...) as the values of science. These values, of truth, honour, rationality, tolerance, and arbitration of disputes were well in evidence in the ancient library, as scholars from all over the world debated on all topics that their imagination would conceive. This great adventure produced some of the most notable breakthrough in the history of human thought» 1 .

Tornou-se claro que a visão assumida para a nova biblioteca era realmente recuperar o espírito da antiga biblioteca, conferindo um papel prioritário ao pluralismo, diversidade e liberdade de expressão: «Not so much to try to collect all the books on the planet - a task arguably beyond the means of even the great Library of Congress with its enormous resources - but to recapture the spirit of openness and dialogue and rationality 
that permeated the ancient library. To encourage the inquisitiveness and the exploration that goes with free inquiry and free speech. To be a space of freedom, where dialogue with the other can take place.»²

Estes propósitos foram desde logo assumidos também quando, em Fevereiro de 1990 (doze anos antes da inauguração), a convite do presidente egípcio Mohamed Hosni Mubarak e da sua esposa Suzanne Mubarak, se reuniram em Assuão, no sul do Egipto, uma série de líderes mundiais, enquanto membros da International Commision for Revival of the Ancient Library of Alexandria, com o objectivo de contribuírem directa e empenhadamente para a prossecução do projecto de construção e dinamização da nova Biblioteca na cidade de Alexandria.

A 12 de Fevereiro assinam a chamada Declaração de Assuão sobre a Bibliotheca Alexandrina ${ }^{3}$, o testemunho e compromisso escrito desta ocasião monumental que consagra os grandes pressupostos inerentes a essa edificação e as primeiras ajudas financeiras para a sua efectiva concretização: os países árabes (Emiratos Árabes Unidos, Arábia Saudita e Iraque) com 65 milhões de dólares e 27 outros países e instituições com uma ajuda que elevou o total até aos 100 milhões de dólares.

Sem ambiguidades, o texto da declaração reconhece que a nova edificação do século XXI teria como referente e motivo inspirador a sua antepassada histórica, construída pelos primeiros Ptolomeus no início do século III a.C.:

«The Bibliotheca Alexandrina will stand as a testimony to a decisive moment in the history of human thought - the attempt to constitute a summum of knowledge, to assemble the writings of the peoples. It will bear witness to an original undertaking that, in embracing the totality and diversity of human experience, became the matrix for a new spirits of a critical inquiry, for a heightened perception of knowledge as a tool and the quest for knowledge as a collaboration process. The Ancient library of Alexandria and its associated Museum gave birth to a new intellectual dynamic. By gathering together all the known sources of knowledge and organizing them for the purposes of scholarly study and investigation, they marked the foundation of the modern notion of the research institute and, therefore, of the university. »

Como salienta o director da Biblioteca, Ismail Serageldin4:

«The New Bibliotheca Alexandrina is dedicated to recapture the spirit of openness and scholarship of the original Bibliotheca Alexandrina. Its mission is to be a center of excellence for the production and dissemination of knowledge, and to be a place of dialogue and 
understanding between cultures and peoples. It is our hope that the New Bibliotheca Alexandrina will be a worthy successor to the Ancient Library of Alexandria. That great Library was a unique ecumenical effort of the human intellect and imagination, and remains engraved in the memories of all scientists and intellectuals to this day. ${ }^{5}$

Da mesma forma, a sua «matriz ideológica» está, assumidamente, desde o texto fundador, impregnada dos ideais defendidos e difundidos pela sua congénere da Antiguidade:

"The Bibliotheca Alexandrina - a link with the past and an opening onto the future will be unique in being the first library on such a scale to be designed and constructed with the assistance of the international community acting through the United Nations system.»

A «ajuda internacional» canalizada para a nova Biblioteca é concebida pela Comissão Internacional como o «reconhecimento da original oferta feita pela antiga biblioteca de Alexandria ao nosso património comum», como consta no parágrafo final da Declaração de Assuão.

Nas palavras do director da BA, esta instituição visa nas próximas décadas quatro grandes objectivos: a) ser a janela do mundo no Egipto; b) ser a janela do Egipto no mundo; c) ser uma instituição líder na era digital e d) ser um vibrante centro de aprendizagem, diálogo e entendimento ${ }^{6}$. Cumpri-los com o decorrer do tempo, através de programas e projectos autónomos ou em parcerias internacionais com outras grandes bibliotecas, universidades, museus, galerias de arte e institutos, é inquestionavelmente assumir a gloriosa herança do passado, adaptando-a às características, exigências e rápidas transformações do nosso tempo.

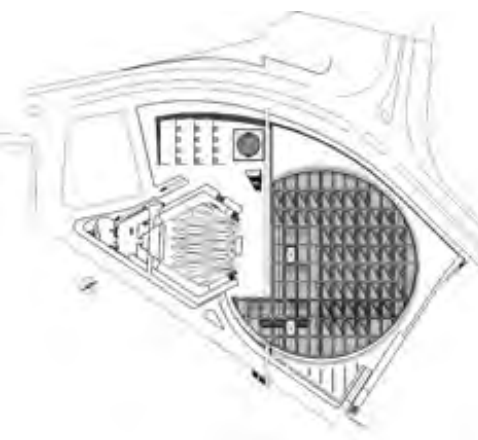

Planta da Bibliotheca Alexandrina (in http://www.bibalex.org/English/aboutus/building/site.htm). 


\section{Do sonho à realidade}

É preciso recuar até ao ano de 1974 para encontrar, de uma forma consistente, a génese da ideia de fazer renascer a antiga Biblioteca de Alexandria. De facto, é nesse ano que o Dr. Lofti Dowidar, presidente da Universidade de Alexandria, lança um vigoroso apelo para a criação de um Comité Internacional que assumisse a responsabilidade pela organização de tal projecto ${ }^{7}$. Além de sustentar teoricamente o projecto, a Universidade de Alexandria deu um claro sinal do seu labor e empenho ao oferecer o terreno para a respectiva edificação ${ }^{8}$.

Muitas ideias necessitam de tempo para crescer e para se robustecer e este foi um desses casos: em 1986, a ideia ganha consistência, com a associação do governo egípcio, da UNESCO e do PNUD (Programme des Nations Unis pour le Développement). A 22 de Outubro de 1987, em Paris, o director-geral da UNESCO, Amadou-Mahtar M'Bow, faz um empenhado apelo à «solidariedade universal»:

«I therefore call on the governments of all States, international governmental and non-governmental organizations, public and private institutions, funding agencies, librarians and archivists, and last but not least, the peoples of all countries to participate, by means of voluntary contributions in cash, equipment or services, in the immense effort undertaken by the Egyptian Government to reconstruct and equip the Library of Alexandria, constitute and preserve its collections and train the requisite personnel.

I call on all intellectuals, artists and writers, historians and sociologists, and on all those whose work it is to inform journalists, columnists, professionals, of the press, radio, television and cinema, to help to develop an awareness by the public in all countries of the universal dimension of the project for the revival of the Library of Alexandria, and to encourage them to contribute to its implementation.

I especially invite the publishers of literary, scientific and artistic books and periodicals the world over to send two copies of each of their publications to the Library of Alexandria, beginning on 1, January, 1988. It is my hope that the contributions will be commensurate with the vast task ahead, and that all those who are concerned for the conservation of the universal documentary heritage and wish to promote the widest possible use thereof by researchers and members of the public all over the world will participate with enthusiasm in the international campaign for the revival of one of the most prestigious institutions in the history of mankind. $)^{9}$ 
Em 1988, o projecto ganha foros de projecto nacional, quando o presidente egípcio cria a GOAL: General Organization for Alexandria Library (Decreto Presidencial $\left.n^{\circ} 523 / 1988\right)^{10}$. Trata-se da criação oficial da Bibliotheca Alexandrina. No ano seguinte, em 1989, a UNESCO e a UIA (International Union of Architects) lançam um concurso internacional, no qual participaram 523 arquitectos de 77 países, com vista à construção do edifício da nova Biblioteca. A vencedora foi a firma norueguesa de arquitectura e paisagística Snohetta, que depois estabeleceu uma joint venture com a empresa egípcia de engenharia Hamza Associates, tendo ambas levado a cabo as tarefas de finalização do design e supervisão da execução do projecto ${ }^{11}$.

Seguiu-se então o histórico encontro de Assuão, de 1990, com a proclamação da sua Declaração que constitui um verdadeiro «turning point» com a entrada em acção dos investidores e do imprescindível suporte financeiro para a implementação do projecto. Ainda assim, nesta fase, os $\$ 100$ milhões constituíam cerca de metade dos estimados $\$ 220$ milhões do custo total do projecto.

Com a participação activa de numerosos políticos e técnicos egípcios e estrangeiros e com a elaboração e aprovação de peças jurídico-legislativas egípcias (por exemplo, a Lei $n^{\circ} 1 / 2001$ e o Decreto Presidencial $n^{\circ} 76 /$ $2001^{12}$ ), o projecto foi, paulatinamente, avançando e aproximando-se da sua efectiva concretização.

A primeira fase dos trabalhos (alicerces e fundações) iniciou-se oficialmente a 15 de Maio de 1995 e terminou a 31 de Dezembro de 1996, através de um consórcio italo-egípcio (Rodio/Trevi-Arab Contractors) que usou as mais avançada tecnologia, tendo custado $\$ 59$ milhões. As operações de reforço da maior parede circular do mundo (160 metros de diâmetro) foi a maior proeza desta fase, a par da colocação dos 600 pilares de betão ${ }^{13}$.

A construção da superestrutura seria levada a cabo, entre 27 de Dezembro de 1996 e 2001, pela joint venture anglo-egípcia Balfour Beatty/ Arab Contractors ${ }^{14}$. Nesta segunda fase da construção gastaram-se $\$ 160$ milhões ${ }^{15}$. O ano de 2002 (16 de Outubro) assinalaria, pois, a inauguração oficial do novo edifício e a conclusão do projecto de fazer reviver a antiga biblioteca de Alexandria.

A concretização deste antigo sonho levou um grande número de estudiosos a considerarem o projecto como «(...) the largest project

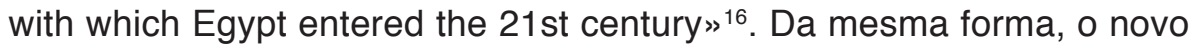


espaço cultural alexandrino «may well be considered the most beautiful and magnificent contemporary building in the world (...). It represents a leap in architectural thinking into the $21^{\text {st }}$ century. The world is now looking forward to the library carrying out its expected cultural role, which is to provide a scientific environment that will reach the highest level available in the whole world. ${ }^{17}$. Coerentemente, em 2004 (27 de Novembro), a Bibliotheca Alexandrina ganhou o Aga Khan Award for Architecture ${ }^{18}$.

\section{A antiga biblioteca de Alexandria: um cadinho intelectual}

A antiga biblioteca de Alexandria foi uma das mais ambiciosas e excitantes aventuras intelectuais na história da humanidade. Criada por acção de Ptolomeu I Sóter (305-285/283 a. C. ${ }^{19}$ ), o diádoco de Alexandre Magno, com a directa colaboração de Demétrio de Falero (350-280 a. C.), discípulo de Aristóteles, o «filósofo do poder», a biblioteca destinava-se a albergar, por indicação real, escritos de todos os povos conhecidos ${ }^{20}$. Como escreve Mustafa El-Abbadi, «This royal command represented a new way of thinking unheard of before that time. This was what made the ancient library of Alexandria distinguished and unique among all the ancient libraries that the world had known, because it became the first universal and encyclopedic library in the whole world» 21 .

Pelo projecto do seu fundador real, a Biblioteca visava a universalidade e devia conservar os escritos de todas as nações: por isso não abrigava apenas os escritos gregos, mas um conjunto completo de arquivos egípcios e de escritos de outras nações. As investigações realizadas dão a impressão que toda a literatura grega se encontrava junta na Biblioteca: livros de Aristóteles, Platão, Ésquilo, Sófocles, Eurípides, Homero (Ilíada e Odisseia), etc. Tornou-se o maior centro de estudos e de cultura grega. De igual modo, continha a herança cultural dos povos do Egipto, Babilónia, Pérsia, Fenícia, Hebreus...

Segundo a lenda, foi na biblioteca de Alexandria que se realizaram projectos como a tradução para grego do Antigo Testamento (hebraico-aramaico), a chamada Versão dos Setenta ou Septuaginta, a obra mais preciosa na história das traduções, essencial a todo o estudo bíblico. Historicamente, sabe-se que a tradução não foi feita toda de uma vez, mas ao longo de vários períodos (ao longo dos séculos III e II a.C.). Certo 
é que o Pentateuco (a Tora) foi traduzido de hebraico para grego, face às necessidades da importante comunidade judaica de Alexandria, já helenizada no fim do século III a. C.

A partir de 295 a. C., a expensas reais, uma plêiade de intelectuais (cientistas, filósofos, poetas, etc.), naturais de Alexandria ou oriundos do mundo mediterrânico, efectuou as suas investigações e estudos nos rolos arquivados nos armaria da antiga biblioteca e conferiu à cidade uma ímpar, durável e muito florescente vida intelectual ${ }^{22}$. Aí se concentrava, segundo o gramático Calímaco de Cirene (315 - 245 a. C., no reinado de Ptolomeu II Filadelfo, época de grande desenvolvimento da biblioteca), a maior colecção de livros até então reunida: 400.000 volumina ou rolos de papiro colados uns aos outros - o equivalente a 35.000 livros modernos.

Além dos meios financeiros dispensados ao projecto de recolecção de papiros ${ }^{23}$ e das compras feitas em Atenas e em Rodes (os dois grandes centros do comércio de livros), os reis ptolomaicos usaram também um curioso processo/ método para a constituição dos seus fundos bibliográficos, sobretudo de obras raras: todos os navios entrados em Alexandria eram revistados e todos os livros que trouxessem eram levados à Biblioteca onde se decidia o que Ihes fazer. Uns eram devolvidos aos proprietários, uma vez que eram obras já existentes nos armaria alexandrinos; outros eram confiscados, sendo entregue aos seus proprietários uma cópia. Os livros assim «adquiridos» eram designados por «livros dos navios», como menciona Galiano no seu Comentário sobre as Epidemias de Hipócrates:

«On raconte que Ptolémée, alors roi d’Egypte, était si fier de ses livres, qu'il avait ordonné que les livres de toute personne qui débarquait lui soient apportés, qu'on en lasse une nouvelle copie sur papyrus, que ce soit la copie qui soit restituée à leur propriétaire, [...], qu'on dépose les livres saisis dans les bibliothèques et qu'on y appose la mention «des navires». [...] Ce Ptolémée mit beaucoup d'ardeur dans l'acquisition de tous les livres anciens comme en témoigne bien anciens comme en témoigne bien le récit de ce qu'il fit aux Athéniens : leur ayant versé une caution de quinze talents en échange des exemplaires de Sophocle, d'Euripide et d'Eschyle pour en faire une unique copie, avant de les rendre immédiatement en parfait état, il les fit copier à grands frais sur le plus beau des papyrus; il garda ce qu'il avait reçu des Athéniens et leur renvoya les copies, les invitant à garder les quinze talents et á accepter, à la place des anciens exemplaires qu'ils lui avaient donnés, les neufs.»24. 
E assim a biblioteca ia-se transformando num imenso «depósito de livros» (apothekai ton biblion) exigindo, naturalmente, «especialistas» dedicados à sua racional organização e adequada manutenção e utilização ${ }^{25}$. No período ptolomaico, entre os seus bibliotecários mais famosos, contou com homens ilustres, de autêntico espírito enciclopédico, como Demétrio de Falero, Zenódoto de Éfeso, Calímaco de Cirene, Eratóstenes de Cirene, Apolónio de Rodes, Aristófanes de Bizâncio e Aristarco de Samotrácia - os sete primeiros bibliotecários (epimeletes), respectivamente, dos reinados de Ptolomeu I a Ptolomeu VI (grosso modo um período de 150 anos).

Como já tivemos ocasião de escrever (Sales, 2006, 70, 71), é justo destacar entre os mais preciosos contributos fornecidos pelos intelectuais de Alexandria ao saber humano, a partir do século III a.C., as primeiras formulações científicas da teoria heliocêntrica, c. de 1800 anos antes de Copérnico, por Aristarco de Samos; o desenvolvimento da medicina, com os estudos de Praxágoras de Cós, Zenão, Mantias, Filino de Cós, Sarapião de Alexandria e Glaucias; a fundação, na primeira metade do século III a.C., da anatomia pelo mais importante dos médicos alexandrinos, Herófilo da Calcedónia, a quem se devem estudos sobre o olho e o nervo óptico, além de ter feito também a distinção entre o cérebro e cerebelo, a separação de tendões e nervos, a descrição do pâncreas e do duodeno (duodénum = dôdekadactylos = «doze dedos»), a descoberta dos ovários femininos, do ritmo do pulso (pulsação sistólica e diastólica) e a sua importância no estabelecimento do diagnóstico médico. Herófilo considerava a saúde o elemento mais importante da vida do Homem, dizendo «sem a saúde, a sabedoria não se desenvolve, o talento não pode manifestar-se, a força não pode entrar em competição, a riqueza torna-se inútil e a razão quase desvalorizada». A sua ideia da circulação sanguínea antecede em cerca de 1700 anos os trabalhos sobre o sistema circulatório do médico britânico William Harvey (1578-1657).

Discípulo de Herófilo da Calcedónia, Erasístrato de Cós (primeira metade do século III a.C.) iniciou a fisiologia, salientando-se no estudo da circulação sanguínea e seus componentes (distinção entre nervos sensitivos e motores, estudo dos vasos sanguíneos e da circulação do sangue, descrição dos pulmões, etc.). «Nutricionista» avant le temps, preocupou-se com o rejuvenescimento dos tecidos do corpo através da alimentação. Criticou o uso de «tratamentos» fortes, como as sangrias e os purgativos usados pela medicina tradicional. 
Também a matemática e a geometria conheceram um progresso notável, podendo mesmo falar-se do estabelecimento das suas bases com homens como Euclides de Alexandria, Apolónio de Perga e Estratão de Lâmpsaco. O primeiro, cerca do ano 300 a.C., redigiu os seus Elementos, em 13 livros, descobrindo as leis da geometria (geometria plana), base dessa ciência até à actualidade. Apolónio de Perga (século III a. C.), por seu turno, destacou-se pelo estudo sobre o valor de $\pi$ e sobre o tratamento global e sistemático das secções cónicas - a elipse, a parábola e a hipérbole (As Cónicas, em 8 livros) -, sendo o seu trabalho uma das obras-primas das matemáticas em grego.

Arquimedes de Siracusa (c. 287-212 a. C.), o maior matemático da Antiguidade, inventor do cálculo integral, autor de numerosos trabalhos de mecânica, inventor de uma bomba hidráulica que ainda hoje se utiliza, celebrizado pelo eureka com que festejou a descoberta da lei da impulsão dos corpos, Héron de Alexandria (meados do século I d.C.) que escreveu vários livros (Mecânicas, Métrica, Catoptrica, Dioptra, Pneumatica, etc.) e inventou uma máquina a vapor, embora sem dela retirar resultados práticos, Ctésibios e Fílon de Bizâncio foram nomes decisivos no âmbito da física e da mecânica. Arquimedes tornou a matemática uma ciência, separando-a de toda a especulação filosófica.

Na geografia e na cartografia merece referência o quarto bibliotecário Eratóstenes de Cirene (276-194 a.C.), o «atleta do espírito» ou «pentaleta» (Levet, 1997, 45, 46), criador da geografia matemática, que, com um erro mínimo de umas centésimas, mediu a circunferência da Terra $(39.602 \mathrm{~km}$ para os reais $40.077 \mathrm{~km}$ ). O grande estóico Posidónio de Apameia ficou também ligado aos estudos geográficos: a ele se deve a formulação da hipótese de que as marés se deviam à atracção lunar.

As competências de Eratóstenes de Cirene fizeram-se também sentir no campo da astronomia. Nesta área, além dele e de Aristarco de Samos (c. 310-230 a.C.) - a quem se deve o cálculo da distância da Terra à Lua em 60 vezes o raio da Terra e a determinação do diâmetro da Lua: igual a 1/3 do terrestre (o valor exacto da relação é 0,27 ) -, ficaram célebres Cónon de Samos (século III a. C.) - denominou uma constelação como «cabeleira de Berenice», em honra da rainha Berenice II, esposa de Ptolomeu III Evérgeta $\mathrm{I}^{26}$-, Dositheos, Hipsicles de Alexandria, Hiparco de Niceia e Claúdio Ptolomeu.

No caso de Hiparco de Niceia (segunda metade do século II a.C.), foi o responsável pelo estabelecimento do ano solar em 365 dias, 5 horas, 55 
minutos e 12 segundos (apenas um erro, por excesso, de 6 minutos e 26 segundos), pela descoberta do fenómeno de precessão dos equinócios, do movimento de oscilação da Terra ou de mutação do seu eixo e pela medição da distância da Terra à Lua (com um erro de menos de 5\%).

Claúdio Ptolomeu (100-180 d.C.), já no século II d.C., portanto, foi autor de Composição Matemática (na Idade Média conhecido como Almagesto), o livro científico sobre a natureza do Universo que maior influência exerceu durante cerca de 1500 anos, uma vez que aí se defendia a teoria geocêntrica que só em 1543 seria definitivamente contrariada por Nicolau Copérnico. O seu catálogo de estrelas comporta a identificação com notável precisão de 1028 estrelas.

Ao longo dos séculos, a biblioteca tornou-se um centro de investigação científica capaz de responder às exigências dos estudiosos que nela trabalhavam. Como menciona Pascale Ballet, todos os cientistas desenvolveram os seus trabalhos em Alexandria e na época ptolomaica puderam «nourrir leurs recherches de textes et de documents conservés à la Bibliothèque ${ }^{27}$.

Os primeiros passos da investigação literária, da História e da história da literatura, da crítica textual, da filologia, da gramática e da lexicografia foram também dados em Alexandria, graças aos trabalhos de homens como Maneton de Sebenitos, Hecateu de Abdera, Zenódoto de Éfeso, Calímaco de Cirene, Filitas de Cós, Eratóstenes de Cirene, Aristófanes de Bizâncio, Aristarco de Samotrácia, Teócrito, Herondas e Dionísio Trácio.

Calímaco de Cirene ficou também eternamente associado à elaboração de um catálogo da biblioteca de Alexandria «em todos os domínios do saber», em 120 rolos: os pinakes. Esta obra chegou-nos muito fragmentada, dividida em secções muito precisas como retórica, direito, epopeia, tragédia, comédia, lírica, história, medicina, matemática, ciências naturais, astronomia, etc. Em cada secção, as obras eram classificadas por ordem alfabética e acompanhadas de uma pequena notícia bibliográfica completada por uma avaliação crítica dos escritos do autor em questão e tábua das matérias ${ }^{28}$.

Os pinakes tornaram-se indispensáveis para os sábios de todo o mundo e serviram de modelo às nomenclaturas do mesmo tipo. Nasceu, assim, pois, a edição científica (atribuição da obra, estabelecimento do texto, divisão em capítulos e criação do sistema diacrítico) e desenvolveu-se a exegese do texto (comentário, índices, constituição de grandes colecções). 
Alguns destes intelectuais foram didaskaloi (preceptores) dos príncipes lágidas (ex.: Filitas de Cós e Estratão de Lâmpsaco foram preceptores do futuro Ptolomeu II; Apolónio de Rodes cumpriu a mesma função junto do futuro Ptolomeu III, como Aristarco de Samotrácia faria junto dos filhos de Ptolomeu VI).

Naturais de Alexandria (a capital dos papiros = livros) ou oriundos de todo o mundo helenístico, quase todos os grandes sábios e estudiosos dos séculos III a I a.C. passaram por Alexandria e com as suas realizações granjearam fama e apreço para a cidade («cidade de todos os saberes»), marcando-a, por assim dizer, para todo o sempre, como capital mundial do saber (suplantando Atenas) e consolidaram para a posteridade a acção e importância da sua biblioteca ${ }^{29}$.

\section{A nova Bibliotheca Alexandrina ou regresso ao futuro}

A estátua colossal de Ptolomeu II Filadelfo (13 m de altura), descoberta nas águas da baía de Alexandria e colocada junto da praça de acesso à entrada principal da BA estabelece um significativo link com o passado. $O$ vasto edifício inclinado da nova biblioteca, de forma cilíndrica, com $160 \mathrm{~m}$ de diâmetro, $305 \mathrm{~m}$ de comprimento da fachada e $85.405 \mathrm{~m}^{2}$ de superfície total, com os seus 11 andares ( 7 dos quais reservados às salas de leitura), qual ícone arquitectónico, dotado do mais moderno sistema de informação bi-alfabética multilingue, conjuga de forma extremamente harmoniosa beleza, futurismo e funcionalidade ${ }^{30}$.

O complexo da Bibliotheca Alexandrina apresenta características estéticas e arquitectónicas, mais ou menos inovadoras, sofisticadas e futuristas, onde uma arquitectura brilhante se conjuga com uma excelente engenharia ${ }^{31}$. Quatro dos pisos da BA encontram-se situados abaixo do nível do solo, enquanto os restantes, acima do solo, acompanham o plano da parte mais elevada do edifício: «Volume, space, light, materials, colors, etc., the entire lexicon of studied architectural criticism is brought to bear on the building, taking it apart and putting it together again both in physical and experiential terms» ${ }^{32}$.

Apesar das suas dimensões, quando visto do exterior, o edifício da BA não choca o observador e denota uma excepcional integração no meio envolvente. Pode dizer-se que não dá a impressão da sua grandeza. À medida que se efectua a transição para o seu interior efectua-se 
também uma gradual transição para uma escala superior, espaçosa, com «respiração» e espectacularidade. As elegantes proporções das colunas, a suave luz natural, o design interno em cascata e a organização das estantes não sugerem nunca um hangar de enormes dimensões; antes, há uma sensação de acolhedora elegância e serenidade.

Do ponto de vista simbólico, a sua forma de disco solar nascente, saindo da água, evoca a antiga mitologia egípcia da época faraónica, onde o Sol era uma importante divindade que, na aurora dos tempos, saiu da água do Nun, o caos aquoso, para reinar no Cosmos. A piscina de água que cerca todo o edifício da BA $\left(4.600 \mathrm{~m}^{2}\right)$, onde foram plantados papiros, pretende justamente sugerir o lençol de água original e o Sol a «flutuar», a sair das águas $^{33}$. O Sol nascente é deveras uma noção apropriada para simbolizar o iniciar de uma nova era de aprendizagem, conhecimento e ecumenismo.

No exterior, a parte mais elevada do edifício ( $33 \mathrm{~m}$ de altura) foi revestida com placas de granito cinzento obtidas em Assuão e está decorada com signos e letras (pictogramas) pertencentes aos alfabetos e às escritas de todo o mundo, num total de 120 linguagens, obra da artista norueguesa Jorunn Sannes que trabalhou com o escultor Kristian Blystad. Todos os painéis usados ( 1 x $2 \mathrm{~m}$ com $15-20 \mathrm{~cm}$ de espessura) foram cortados à mão.

A cobertura, em parte fortemente inclinada, é formada por painéis de vidro especiais, assentes sobre uma estrutura de vigas em aço, que permite canalizar para o interior das salas de leitura uma luz natural difusa controlada por um complexo sistema de reflectores semelhantes a guarda-chuvas invertidos.

Sem querermos ser exaustivos na descrição das suas múltiplas funcionalidades, importa salientar alguns componentes e números associados a esta construção. Desde logo, é preciso ter presente que o complexo da Bibliotheca Alexandrina não se restringe à biblioteca; inclui a biblioteca principal, obviamente, mas também a biblioteca Taha Hussein para invisuais, a biblioteca para jovens, a biblioteca infantil, a biblioteca multimédia, o Museu de Antiguidades, o Museu dos Manuscritos, o Museu da Ciência, o Planetário, a Escola Internacional para os Estudos de Informação, o Instituto de Caligrafia e Escrita, o Laboratório de Conservação e Restauro, o Centro de Documentação e Investigação e o Centro de Conferências, além das áreas e salas de serviços, de exposição e de fins variados ${ }^{34}$.

Em relação aos 7 andares dedicados a salas de leitura, a disposição do espaço obedece a uma intencional «arrumação do saber»: os quatro pisos inferiores (B4, B3, B2 e B1, respectivamente, de baixo para 
cima), são consagrados à Filosofia, à Religião, à História, à Geografia, à Caligrafia e aos mapas (B4: «raízes do conhecimento»); à Literatura, à Poesia, à Biblioteca Taha Hussein para invisuais (B3); à Arte, à Cultura e à Biblioteca especializada de materiais audiovisuais (B2); a exposições, a galerias e referências genéricas a publicações europeias e das Nações Unidas, além dos três Museus das Antiguidades, dos Manuscritos e de História da Ciência (B1). O piso de entrada (E) é consagrado às Ciências Sociais, Direito e arquivos de Internet. Os dois pisos superiores (F1 e F2, respectivamente) são dedicados, o primeiro, às Ciências Naturais e o último, o mais elevado ( $7^{\circ}$ andar), às Novas Tecnologias ${ }^{35}$.

Estes vários andares e as suas respectivas salas de leitura fornecem 2000 lugares de leitura em simultâneo e apresentam condições para albergar os almejados 8 milhões de volumes, 4000 periódicos, 50.000 mapas, 50.000 manuscritos e livros raros e 50.000 diversos materiais audiovisuais e multimédia ${ }^{36}$.

O espaço dedicado à biblioteca principal ocupa $13.625 \mathrm{~m}^{2}$, além dos $3.930 \mathrm{~m}^{2}$ das bibliotecas especializadas. Os Museus dispõem de 2.055 $\mathrm{m}^{2} \mathrm{e}$ as galerias de arte e de exposições de $4.540 \mathrm{~m}^{2}$, a que há que juntar igualmente os $10.900 \mathrm{~m}^{2}$ das áreas de serviços adstritos à colossal logística de organização e de funcionamento internos. O complexo compreende ainda várias salas de conferências $\left(3.020 \mathrm{~m}^{2}\right)$ : Grande (1700 lugares), Central (290 lugares), Ocidental (288 lugares), Oriental (286 lugares) e o Auditorium (100 lugares). O planetário dispõe de 99 lugares.

A Bibliotheca Alexandrina é muito mais do que um simples ícone arquitectónico do Egipto moderno; é mais do que o mais bonito e magnífico edifício contemporâneo do mundo moderno; é mais do que apenas o maior projecto cultural com que o Egipto entrou no século XXI.

Independentemente das características estéticas e arquitectónicas patenteadas pelo conjunto de edifícios que compõem a nova biblioteca de Alexandria e da gigantesca logística inerente ao seu funcionamento e manutenção, a BA vale essencialmente pela mensagem que lhe está inerente: torná-la, como acontecia com a da Antiguidade, um observatório intelectual de encontro de culturas e, ao mesmo tempo, de estudo e de compreensão dos valores profundos das culturas em presença. 
Fazendo dos documentos scripto, audio e video a base de registo e suporte do saber humano, aberta a investigadores de todos os quadrantes geográficos e ideológicos, a nova Biblioteca pretende objectivamente enfatizar o papel que a cultura pode desempenhar no estabelecimento e na consolidação de plataformas de conhecimento e de entendimento entre as diferentes culturas e civilizações.

Esta ideia de uma biblioteca universal como principal factor de promoção do diálogo e do entendimento interculturais é, nos tempos actuais, extremamente feliz. O projecto é, naturalmente, ambicioso, difícil e, até, de certa forma, utópico, sobretudo quando posto em flagrante contraste com a situação concreta da actual política internacional de "choque de civilizações», particularmente depois do 11 de Setembro de 2001. Mas se pensarmos na utopia e no sonho como motores de novos comportamentos e vontades, então talvez se deva reconhecer que se trata de uma possibilidade a não enjeitar. E, no fundo, se as «soluções» económicas, políticas, ideológicas e militares experimentadas ao longo de séculos fracassaram, nalguns casos a toda a linha, restará algo mais além da cultura para criar um espaço de liberdade? Não será o conhecimento, realmente, o melhor meio de preservar as identidades, recuperar a esperança e assegurar a paz?

\section{ANEXOS}

\section{Doc.1. ASWAN DECLARATION ON THE BIBLIOTHECA ALEXANDRINA}

At the beginning of the third century before our era, a great enterprise was conceived in ancient Alexandria, meeting-place of peoples and cultures: the edification of a Library in the lineage of Aristotle's Lyceum, transposing Alexander's dreams of empire into a quest for universal knowledge.

On the eve of the third millennium and under the patronage of President Mohamed Hosny Mubarak, the Government of the Arab Republic of Egypt is seeking, in cooperation with UNESCO and with the financial support of UNDP and other public and private sources, to revive the Ancient Library of Alexandria by restating its universal legacy in modern terms.

The Bibliotheca Alexandrina will stand as a testimony to a decisive moment in the history of human thought - the attempt to constitute a summum of knowledge, to assemble the writings of the peoples. It will bear witness to an original undertaking that, in embracing the totality and diversity of human experience, 
became the matrix for a new spirits of a critical inquiry, for a heightened perception of knowledge as a tool and the quest for knowledge as a collaboration process.

The Ancient library of Alexandria and its associated Museum gave birth to a new intellectual dynamic. By gathering together all the known sources of knowledge and organizing them for the purposes of scholarly study and investigation, they marked the foundation of the modern notion of the research institute and, therefore, of the university.

Within this haven of learning, the arts and sciences flourished for some six centuries alongside scholarship.

The classification and exegesis of the classical literacy canon nourished the poetic wit of Callimachus and the pastoral muse of Theocritus. Study of the theories of the masters of Greek thought, informed by the new Alexandrian spirit of critical and empirical inquiry, yielded major insights and advances in those branches of science associated with the names of Euclid, Herophilus, Erastosthenes, Aristarchus, Ptolemy, Strabo, Archimides and Heron.

The achievements of Alexandrian science, lost to the West for over a millennium before their partial recovery via Constantinople and classical Arabic and Islamic cultures, were to be instrumental in launching the European Renaissance on its quest for new worlds. In this and as the transmitter of Greek civilization in general, the Ancient Library of Alexandria survives as a vital link in a living tradition.

On the site of the palace of the Ptolemies, the new Alexandrina will give modern expression to an ancient endeavor. A splendid contemporary design for the Library has already been adopted through an international architectural competition. Detailed plans exist for a facility embodying the latest computer technology and serving as a public research library. Conceived in the framework on the World Decade for Cultural Development, this institution will be open to researchers not only from the Mediterranean countries but from all over the world. Its specialized collections will relate to the Egyptian, other middle-Eastern and Greek civilizations, the birth of Coptic Christianity and the Islamic heritage, with special emphasis on the history of sciences and on works likely to have been contained in the original Library. Subsequently the Library will expand to cover other disciplines, in keeping with its universal vocation. It will thereby contribute both to the development of the region in which it is situated and to the understanding of that region throughout the world.

The Bibliotheca Alexandrina - a link with the past and an opening onto the future will be unique in being the first library on such a scale to be designed and constructed with the assistance of the international community acting through the United Nations system.

We, the members of the International Commission for the revival of the Ancient Library of Alexandria, meeting at its inaugural session in Aswan in February 1990 under the chairmanship of Mrs. Suzanne Mubarak, pledge our wholehearted 
support and commitment to this project and reiterate to this end the appeal made by the Director-General of UNESCO in 1987.

We call upon all governments, international governmental and non-governmental organizations, public and private institutions, funding agencies, librarians and archivists, and the people of all countries to participate, by means of voluntary contributions of all kinds, in the effort initiated by the Egyptian Government to revive the Library of Alexandria, to assemble and preserve its collections, to train the necessary staff to ensure the Library's functioning.

We call on scholars, writers and artists and all those whose task it is to inform through the written and spoken word to help to generate awareness of the international project for the revival of the Library of Alexandria and support for this historic venture.

Finally, we urge all governments to donate to the Bibliotheca Alexandrina such works in their possession as will help to constitute and enhance the Library's collections, in recognition of the unique gift made by Ancient Library of Alexandria tour common heritage.

\section{Signatories}

- Madame Susanne AGNELLI, Sénateur, Sous-Secrétaire aux affaires étrangères (Italie) ;

- Her Majesty Queen Noor AL-HUSSEIN of Jordan;

- Mr Yahya Bin Mahfoudh AL-MANTHERI, Minister of Education and Youth (Sultanate of Oman);

- His Highness Sheikh Zayed Bin Sultan AL-NAHYAN, President of the United Arab Emirates;

- His Royal Highness Prince Turki Ibn Abdal-Aziz AL-SAUD, Founder and President of the Arab Student Aid International (ASAI) (Saudi Arabia);

- Dr Daniel BOORSTIN, Historian, Librarian of Congress Emeritus (United States);

- Lord BRIGGS, Provost, Worcester College, Oxford (United Kingdom);

- Mrs Gro Harlem BRUNDTLAND, Member of Parliament (Norway);

- Son Altesse Sérénissime la Princesse CAROLINE de Monaco;

- Mr Hans-Peter GEH, President of the International Federation of Libray Associations and Institutions (IFLA) (Federal Republic of Germany);

- Mr Abdul-Aziz HUSSAIN, Adviser to His Highness the Amir of Kuweit ;

- Professor Dmitri Sergeevich LIKHACHEV, Academician (USSR);

- The late Madame Melina MERCOURI, Membre du Parlement (Grèce);

- The late Monsieur François MITTERAND, Président de la République française;

- Mrs Susan MUBARAK (Egypt);

- Sa Majesté la Reine SOFIA d' Espagne; 
- Mr Ahmed Fathi SOROUR, Minister of Education, Chairman of the General Organization of the Alexandria Library (GOAL) (Egypt);

- Mr José Israel VARGAS, Former Chair of the Executive Board of Unesco (Brazil).

\section{Doc. 2. LAW No.1 FOR THE YEAR 2001}

Concerning the Library of Alexandria

In the name of the People

The President

The People's Assembly has enacted the following law and we have hereby issued it:

\section{Article 1}

The Library of Alexandria is a public juridical person headquartered in the city of Alexandria, attached to the President. It is an Egyptian center of cultural radiance, a beacon for thought, culture and science, encompassing all the products of the human mind in all languages, from all cultures, ancient and modern.

\section{Article 2}

The Library comprises the Library, the Planetarium and the Conference Center and it is to have the following cultural and scientific institutions established within it: International School for Information Studies (ISIS), Center Documentation and Research, Science Museum, Calligraphy Institute, Manuscripts Museum, Center for the Preservation of Rare Books and Documents;

Other cultural or scientific institutions may be added or established by a decree of the President of the Republic, and the President of the Republic shall by decree define the Legal status of the institutions mentioned in this article.

\section{Article 3}

The Library shall undertake all activities that serve its mission, and undertake all actions related to so doing, by:

- Obtaining studies, books, periodicals, manuscripts, papyri and other items that are related to the Egyptian civilization in its different ages, and related to the scientific, intellectual and cultural heritage of the countries of the world.

- Collecting originals or copies of manuscripts that represent the intellectual achievements of the Islamic and Arab World in ancient and modern languages. 
- Collecting materials connected to the biographies and achievements of eminent figures in the realms of thought, science, politics and religion throughout human history.

- Undertaking studies connected to the historical, geographic, cultural and religious aspects of the Mediterranean region, the Middle East, Egypt and Alexandria, in particular.

\section{Article 4}

The President of the Republic shall determine by decree the manner in which the Library shall be supervised and administered, and the method in which its administrative and financial affairs shall be run, in a manner appropriate with the nature of its activities to enable it to achieve its mission, and this without being constrained by the administrative regulations stated in any other law.

\section{Article 5}

The resources of the Library shall be comprised of:

- The allocations provided for it by the State.

- The support, gifts, donations, bequests, and financial contributions from internal or external sources.

- The loans that will be secured for its benefit.

- The income it shall receive for its services and the returns on the invested funds.

Any other sources that may be legally allocated to the Library.

\section{Article 6}

The Library shall have an independent budget, and its financial year shall start and end with the financial year of the government's budget.

The Library shall have a separate account in the Central Bank of Egypt or in a commercial bank subject to the approval of the Minister of Finance.

It shall deposit in a said account the proceeds of its resources. The surpluses in that account shall be carried forward from one financial year to the next.

\section{Article 7}

Within the limits of its official use and requirements, the Library and its affiliated bodies shall be exempt from general taxation of its surpluses and the revenues of its current activities, and from all charges for registration and declaration, and from customs duties on its imports of scientific material. 


\section{Article 8}

Presidential Decree No.523 for 1988, establishing the General Organization for the Library of Alexandria, shall remain operative in all matters that do not contravene this Law, until the Presidential Decree mentioned in Article Four of this Law shall be enacted. Thereupon, all the assets, rights and obligations of the abolished Organization shall revert to the Library.

\section{Article 9}

This Law shall be published in the Official Journal and shall enter into force the day following its publication. Set the official seal of the State, and is to be executed as a law of the State. Issued at the Presidency of the Republic on 17 Dhul Hijja 1421 AH, 12 March 2001.

(signed)

Hosni Mubarak

\section{Doc. 3. PRESIDENTIAL DECREE No. 76 FOR THE YEAR 2001}

Regarding the organization of the supervision of the Library of Alexandria and the manner of its management and the conduct of its financial and administrative affairs, The President, after reviewing the Constitution and Law No. 1 of 2001 concerning the Library of Alexandria, and the agreement of the Council of Ministers, has decreed:

\section{Article 1}

The Library of Alexandria is a public juridical person headquartered in Alexandria and attached to the President.

\section{Article 2}

The Management of the Library of Alexandria

The Library of Alexandria shall be managed by:

(a) A Council of Patrons;

(b) A Board of Trustees;

(c) The Director-General. 


\section{Article 3}

The Council of Patrons

The Council of Patrons is composed of a number of prominent persons from different parts of the world, numbering not less than eight and no more than twenty-four, to be chosen by invitation of the President of the Republic, and one of these persons shall be the Director-General of the UNESCO.The President or his designate shall be the Chair of the Council of Patrons, and the Minister of Higher Education shall be the Secretary of the Council.

The Council shall have the responsibility of supporting the Library and following up on its activities and shall express its views on the direction of such activities. The Council shall meet once every three years at the invitation of its Chair.

\section{Article 4}

The Board of Trustees

The Board of Trustees shall be composed of a number of eminent persons of scientific and intellectual standing or international experience, among Egyptians and non-Egyptians. They shall number no less than fifteen and no more than thirty; of whom five shall be ex-officio members of the Egyptian Government, namely: the Minister of Higher Education, the Minister of Culture, the Minister of Foreign Affairs, the Governor of Alexandria and the President of the University of Alexandria. The Chair of the Council of Patrons shall be the Chair of the Board of Trustees, and the said Chair shall choose a member of the Board to act for the Chair in case of the Chair's absence. The first Board of Trustees shall be named by a decree issued by the President of the Republic, and for the non-ex-officio members of that Board, the duration of their membership shall be two years. After that period, the Board membership shall be renewed by a third every year. Other than this first Board, the non-ex-officio members of the Board shall be appointed by a decision of the Board of Trustees on nomination of one of the members. Membership shall be for three years, renewable for one additional term.

The Board of Trustees is the decision-making power on the matters of the Library of Alexandria, and will be responsible for defining its general policies, for the administration and planning of its activities and for the establishment of its administrative and financial regulations.

The Board shall meet once a year and can hold exceptional (additional) meetings by invitation of its Chair or at the request of no less than half of its members.

The meeting of the Board shall be deemed legal if a majority of the members are in attendance, and decisions shall be by simple majority of those present. In case of equal votes, the side with the presiding Chair's vote will prevail. The Board may constitute from its membership committees to which it may assign the execution of specific tasks, or the preparation of particular studies or the execution of research. The Library of Alexandria shall bear the cost of travel and honoraria associated with the attendance of the Board meetings or the committees established by it. 


\section{Article 5}

The Director

The Board of Trustees shall appoint the Director of the Library for a period of five years, renewable, and shall determine the Director's compensation. This appointment shall be by a decision of the Board supported by two-thirds of its attending members, and it is essential that the candidate be a person of international standing, wide culture, and managerial and technical competence.

The Director is the Chief Executive Officer of the Library of Alexandria, and is entrusted with the execution of the policies established by the Board of Trustees. The Director shall prepare the agenda for the Board meetings, and shall attend the meetings, but shall not vote on decisions before the Board.

The Director shall be the head of the staff, and shall appoint the staff, promote them and terminate their services, in accordance with the legal statutes they will serve under. The Director shall be the legal representative of the Library of Alexandria before the judiciary and in its dealings with others.

\section{Article 6}

Administrative and Financial Regulations and Statutes for Staff

The Director shall prepare the Administrative and Financial Regulations and Statutes for Staff in a manner that is compatible with the nature of the Bibliotheca Alexandrina's activities and that would enable it to execute its mission without being bound by other administrative statutes specified in other laws, and these regulations and statutes shall be submitted to the Board of Trustees for their approval. Upon approval by the Board of Trustees, the Statutes for Staff shall be the legal statutes that shall govern the relationship between the Library of Alexandria and its staff.

\section{Article 7}

Budget and Auditors

The Library of Alexandria shall have an independent budget and the surplus shall be carried over from one fiscal year to the next. Without prejudice to the supervision of the Government Accounting Office, the Board of Trustees shall appoint the external auditors and shall receive their reports.

\section{Article 8}

This Decree shall be published in the Official Journal and shall be operative from the date of its publication.

(Signed)

Hosni Mubarak 


\author{
Issued at the Presidency of the Republic \\ On 25 Dhul Hijja $1421 \mathrm{AH}$ \\ corresponding to 20 March $2001 \mathrm{AD}$
}

\title{
Notas
}

(1) Serageldin 200225 .

(2) Ibid., 26.

(3) Vide o texto integral desta Declaração no final deste artigo. A Declaração de Assuão foi assinada pelos seguintes estadistas: Susanne Agnelli, senadora e sub-secretária dos Negócios Estrangeiros de Itália; a rainha Noor Al-Hussein da Jordânia; Yahya Bin Mahfoudh Al-Mantheri, ministro da Educação e da Juventude do Sultanato de Oman; Sua Alteza Sheikh Zayed Bin Sultan Al-Nahyan, presidente dos Emirados Árabes Unidos; Sua Alteza Real, o príncipe Turki Ibn Abdal-Aziz Al-Saud, fundador e presidente da ASAI (Arab Student Aid International), da Arábia Saudita; Daniel Boorstin, historiador e bibliotecário do Congresso, dos Estados Unidos da América; Lord Briggs, reitor do Worcester College, Oxford, do Reino Unido; Gro Harlem Brundtkland, membro do Parlamento norueguês; Sua Alteza Sereníssima, a princesa Carolina, do Mónaco; Hans-Peter Geh, presidente da International Federation of Library Associations and Institutions (IFLA), Alemanha; Abdul-Aziz Hussain, conselheiro de Sua Alteza o Amir do Koweit; Dimitri Sergeevich Likhachev, académico russo; Melina Mercouri, membro do Parlamento grego; François Mitterrand, presidente da República Francesa; Susan Mubarak, representante do Egipto; Sua Majestade, a rainha Sofia de Espanha; Ahmed Fathi Sorour, ministro da Educação, presidente da General Organization of the Alexandria Library (GOAL), do Egipto; José Israel Vargas, ex-presidente do Comité Executivo da Unesco, do Brasil (Cf. http://www.touregypt.net/library/revivald.htm).

(4) Nomeado pelo presidente egípcio no final de Maio de 2001, o actual bibliotecário Ismail Serageldin é, no fundo, com dois milénios de permeio, herdeiro dos grandes nomes do passado que exerceram o mesmo cargo na Antiguidade, como Zenódoto de Éfeso, Apolónio de Rodes, Eratóstenes de Cirene, Aristófanes de Bizâncio ou Aristarco de Bizâncio.

(5) http://www.bibalex.org/ENGLISH/aboutus/rebirth/mission.htm (17/01/2010; 19.37).

(6) Cf. Ibid. 11, 27-29.

(7) A par do presidente da Universidade de Alexandria, é de destacar a acção dedicada de Mustafa El-Abbadi, professor de História Grega e Romana da Faculdade de Artes, que foi um dos grandes promotores nos anos 70 do século XX do projecto de recriação da antiga biblioteca (Cf. Hawass 2002 7).

(8) Para um grande número de arqueólogos, o terreno cedido, onde a Bibliotheca Alexandrina viria a ser construída, corresponde ao local da antiga Biblioteca: el-Selsela, no Brucheion, o antigo bairro real da cidade ptolomaica. Segundo Estrabão (XVII, 1, 8) era aí que se localizava o Museu-Biblioteca, mesmo em frente ao palácio real ptolomaico (a nordeste do Cabo Lochias): «O Museu faz também parte dos edifícios reais e compreende um grande corredor coberto, uma exedra com assentos e um grande edifício onde se encontra a sala comum onde os sábios, os membros do Museu, tomam as suas refeições». As escavações no local iniciaram-se em Maio de 1993 e prolongaram-se até 1995, numa área de $20.000 \mathrm{~m}^{2}$, tendo sido encontrados traços do antigo bairro real, incluindo importantes e artisticamente elaborados mosaicos, nomeadamente um, incompleto, em mármore colorido e cubos de calcário, em estilo opus vermiculatum no interior e opus tesselatum na bordadura exterior, com $3,25 \mathrm{~m} \times 2,75 \mathrm{~m}$, representando um ginásio ou um banho público, onde, ao lado de uma fonte, dois gladiadores nus combatem, um branco, grego, e outro negro, provavelmente africano, e outro, com $1,35 \mathrm{~m} \times 1,35 \mathrm{~m}$, também em mármore colorido com cubos de calcário, representando um cão ao lado de um invertido askos (capacete grego em bronze). Hoje estes mosaicos encontram-se no museu 
integrado na nova biblioteca de Alexandria, com os números de inventário 32045 e 32044, respectivamente (Cf. Fatah 2002 51-54; http://antiquities.bibalex.org/Collection/Detail.aspx?collection=43\&a $=858 \&$ lang=en e http://antiquities.bibalex.org/Collection/Detail.aspx?collection=43\&a=859\&lang=en).

(9) http://www.bibalex.org/English/aboutus/rebirth/appeal.htm.

(10) Cf. O texto do Decreto Presidencial e o logótipo da GOAL em http://www.bibalex.org/English/ aboutus/rebirth/goal.htm.

(11) Ibid. 11.

(12) Vide os 9 artigos da Lei ${ }^{\circ} 1$ em http://www.bibalex.org/ENGLISH/aboutus/orgnization/law1. htm e os 8 artigos do Decreto Presidencial em http://www.bibalex.org/English/aboutus/orgnization/decree.htm. Vide Anexos no final deste texto. A presidente do BOT (Board of Trustees - art $^{\circ}$ 4 da Lei $n^{\circ} 1$ ) é a primeira dama egípcia, Suzanne Mubarak. Com estes dois documentos legais, a Biblioteca tornava-se a única instituição dotada dos meios e do potencial para realmente implementar a visão associada à sua designação.

(13) Ibid. 45.

(14) http://www.arabcont.com/english/projects/projects. aspx?sec id=1\&subsec id=2.

(15) Ibid. 45.

(16) El-Abbadi 200248.

(17) Ibid. 48.

(18) Cf. Serageldin 2007 8, 46.

(19) A data de 283 a. C. refere-se objectivamente ao ano em que Ptolomeu I morreu, mas o próprio rei dois anos antes (desde 285 a. C.) associou o seu filho e sucessor, Ptolomeu II, como co-regente (Cf. Chauveau 1997 163-164).

(20) Demétrio de Falero era membro da escola peripatética e fora antigo governador de Atenas (317-307 a. C.). Expulso do poder em Atenas, em 297 a. C., refugiou-se na corte de Ptolomeu I, de quem se tornou fiel conselheiro.

(21) El-Abbadi 2002 47, 48.

(22) Id. 1998 116. Não podemos esquecer que, a par da biblioteca, também o Museu, «templo das Musas» (onde a biblioteca estava integrada), foi fundado por Ptolomeu I Sóter, tendo ficado associado ao desenvolvimento da ciência no período helenístico: "Les hommes de science trouvèrent tous à Alexandrie aide, protection et encouragements, et ils furent nombreux à se réunir dans cette ville pour travailler et apprendre auprès de maîtres illustres, avant de devenir eux-mêmes des chercheurs et des formateurs. Le pouvoir politique leur fournissait des conditions de travail très favorable, mais les grandes acquisitions scientifiques se furent surtout au IIle avant J.-C., avec des noms prestigieux, passés à la postérité et que tout le monde connaît, dans les domaines des mathématiques, de l'astronomie et de la médicine.» (Argoud 1998 131). Entre os seus primeiros directores encontraram-se Demétrio de Falero e Estratão de Lâmpsaco, ambos discípulos de Aristóteles e defensores e promotores do saber universal que o seu mestre proclamara (Cf. Mossé 1992 83-92; Bernand, 1996 112-118; El-Abbadi 1992 112).

(23) Com Ptolomeu I, época inicial do projecto, falamos de 100.000 rolos, para um máximo, em meados do século I a.C., de 700.000 .

(24) Apud in Ballet 1999120.

(25) Diodoro (I, 46.8; 87.1-5; 88.4) refere ainda um outro processo usado por Ptolomeu I para aumentar os exemplares da Biblioteca: segundo ele, o faraó egípcio teria pedido ao sacerdote Maneton, a 
Hecateu de Abdera e a outros intelectuais que reunissem papiros egípcios dos santuários egípcios.

(26) Cerca de 243 a. C., quando o seu marido partiu em campanha militar para a Mesopotâmia, Berenice II prometeu os seus longos cabelos à deusa Afrodite se esta zelasse pelo seu marido e o trouxesse de volta ao Egipto, são e salvo. Uma vez que tal aconteceu, a rainha cortou a sua cabeleira e colocou-a no altar de Afrodite. No dia seguinte, porém, a cabeleira havia desaparecido. Conhecedor desta história, Cónon de Samos afirmou que Afrodite ficara tão encantada com a oferenda que a levara para o céu. Daí a designação que deu à constelação.

(27) Ballet 1999116.

(28) Os funcionários encarregues do agrupamento e classificação dos rolos e volumes eram os hyperetae.

(29) Em abono da verdade, é necessário referir que no reinado de Ptolomeu III Evérgeta I dá-se o estabelecimento de uma «biblioteca filha» no Serapeum de Alexandria (ou «biblioteca filha de Rakotis»), para onde foram transferidos os volumes menos necessários ou as cópias da megale bibliotheke, "Grande Biblioteca». A biblioteca do Serapeum seria destruída pelos cristãos, através de um incêndio, em 391 d.C., na época do imperador Teodósio e do patriarca de Alexandria, Teófilo (Cf. Bernand 1996 121). A «biblioteca-mãe» desaparecera já, havia muito, após o incêndio de 48-47 a. C., na época da presença-acção de Júlio César na capital lágida.

(30) http://www.unesco.org/comnat/france/alexandrie.htm

(31) O custo total das obras fixou-se nos $\$ 176$ milhões (cerca de 200 milhões de $€=40$ milhões de escudos).

(32) Serageldin 200235.

(33) A relação entre o edifício circular da Biblioteca e o esférico do Planetário é, pelas dimensões, simbolicamente também, a relação entre o Sol e a Lua.

(34) Ibid. 45.

(35) Cf. Ibid. 37, 38; Id. 2007, 19, 21. Plantas dos vários pisos da Bibliotheca, com as indicações das respectivas áreas e espaços, e alçados das fachadas este e oeste podem ser vistos em Ibid. 47-59.

(36) À data da sua abertura em 2002, a biblioteca possuía, 200.000 volumes, 1500 periódicos, 2000 mapas, 10.000 materiais audiovisuais e multimédia e 10.000 manuscritos e livros raros (Cf. Id. 2002 45).

\section{Bibliografia}

- AAVV (1998), L'ABCdaire d'Alexandrie. Paris: Flammarion.

- G. ARGOUD (1998), «Science et ingénieurs alexandrins», in La gloire d'Alexandrie, Catalogue générale de l'exposition «La gloire d'Alexandrie» (Paris, 7 mai-26 juillet 1998), Paris: Paris-Musées, 118-133.

- S. Bakhoum (1995), “Les édifices alexandrins d'après les documents monétaires», in Alexandrie. Lumière du Monde Antique. Les Dossiers d'Archéologie, n² 201, Mars 1995, Dijon: Éditions Faton, 2-11.

- P. BALLET (1999), La vie quotidienne à Alexandrie. 331-30 avant J.-C. Paris: Hachette.

- A. BERNAND (1995), Alexandrie des Ptolémées. Paris: CNRS.

(1996), Alexandrie, la Grande. Paris: Hachette. 
- P. BING (1998), “La culture littéraire d'Alexandrie au Ille siècle avant J.-C.», in La Gloire d'Alexandrie, Catalogue générale de l'exposition «La gloire d'Alexandrie» (Paris, 7 mai-26 juillet 1998), Paris: Paris-Musées, 133-135.

- N. BONACASA (1995), «Alessandria capitale e l'Egitto dei Tolemei», in A. Di Vita, C. Alfano, eds., Alessandro Magno: Storia e Mito. Palazzo Ruspoli, Roma. 21 dicembre 1995 - 21 maggio 1996, Milão: Leonardo Arte, 67-79.

- L. CANFORA (1988), La véritable histoire de la bibliothèque d'Alexandrie. Paris: Editions Desjonqueres.

- M. CHAUVEAU (1997), «Ères nouvelles et corégences en Égypte ptolémaïque», in Akten des 21. Internationalen Papyrologenkongresses, Berlin 1995. Archiv für Papyrusforschung, Beiheft 3, Leipzig/Stuttart: B. G. Tenbner, 163-171.

- M. EL-ABBADI (1998), "La bibliothèque d'Alexandre», in La gloire d'Alexandrie, Catalogue générale de l'exposition «La gloire d'Alexandrie» (Paris, 7 mai-26 juillet 1998), Paris: Paris-Musées, 112.

(2002), «The Ancient Library: Intellectual beacon», in Zahi Hawass, ed., Bibliotheca Alexandrina. The Archaeology Museum, Zamalek: The Supreme Council of Antiquities, 47-49.

- J. -Y. EMPEREUR (1993), «Alexandrie, ville grecque en terre égyptienne», in Le Grand Atlas de l'Art, Paris: Encyclopaedia Universalis, 154-155.

- __ (1998a) Alexandrie redécouverte. Paris: Fayard/ Stock.

- __ (1998b), Le phare d'Alexandrie. Paris: Découvertes Gallimard.

-__ (2002), Alexandrie. Past, present and future. London: Thames \& Hudson.

(2003), «Du nouveau sur la topographie d'Alexandrie», in Comptes rendus de l'Académie des Belles Lettres, fascicule 3, Paris, 921-933.

- A. A. FATAH (2002), «The Excavations of the Site of Bibliotheca Alexandrina», in Zahi Hawass, ed., Bibliotheca Alexandrina. The Archaeology Museum, Zamalek: The Supreme Council of Antiquities51-62.

- P. M. FRASER (1972), Ptolemaic Alexandria, vols. 1 e II. Oxford: The Clarendon Press.

- F. J. GÓMEZ ESPELOSíN (1997), «Alejandría, la ciudad de las maravillas», in Ciudades del mundo antiguo, Madrid: Ediciones Clásica, 63-81.

- F. J. GÓMEZ ESPELOSíN, Antonio Pérez Largacha (1997), «La Biblioteca ideal», in Egiptomania, Madrid: Alianza Editorial, 74-91.

- M. HAAG (2004), Alexandria Illustrated. Cairo, New York: The American University in Cairo Press.

- Z. HAWASS (ed.) (2002), Bibliotheca Alexandrina. The Archaeology Museum. Zamalek: The Supreme Council of Antiquities.

- C. JACOB (1992), «Un athlète du savoir: Ératosthêne», in AAVV, Alexandrie II/e siècle av. J.-C. Tous les savoirs du monde ou le rêve d'universalité des Ptolémées, Paris: Éditions Autrement 127.

- La gloire d'Alexandrie (1998), Catalogue générale de l'exposition «La gloire d'Alexandrie» (Paris, 7 mai-26 juillet 1998), Paris: Paris-Musées.

- J. LALLOT (1992), «Zénodote ou l'art d'accommoder Homère», in AAVV, Alexandrie IIfe siècle av. J.-C. Tous les savoirs du monde ou le rêve d'universalité des Ptolémées, Paris: Éditions Autrement, 100-113. 
- J.-P. LEVET (1997), «Un savant d'Alexandrie: Eratosthene (IIIe siècle avant J.-C.). Un regard d'aujourd'hui», Le Monde Copte. Revue trimestrielle de la culture copte, $\mathrm{n}^{\circ \mathrm{s}} 27-28$, Paris: S.I.P. E., 45-58.

- J. G. MANNING (2009), The last pharaohs. Egypt under the Ptolemies, 305-30 BC. Princeton: Princeton University Press.

- J. MÉLĖZE-MODRZEJEWSKI (1996), «Alexandrie entre l'Égypte et la Grèce», in AAVV, L'Égypte ancienne, Paris: Éditions du Seuil, 189-199.

- Cl. MOSSE (1992), «Démétrios de Phalère: un philosophe au pouvoir», in AAVV, Alexandrie $I I /{ }^{e}$ siècle av. J.-C. Tous les savoirs du monde ou le rêve d'universalité des Ptolémées, Paris: Éditions Autrement, 83-92.

- J. H. C. NEWMAN (1997), «L'école d'Alexandrie, précurseur de l'université moderne», Le monde copte, nos 27-28, juin 1997, 129-132.

- J. das C. SALES (2005), Ideologia e propaganda real no Egipto Ptolomaico (305-30 a.C.). Lisboa: Fundação Calouste Gulbenkian. (2006), "A condição multicultural da antiga cidade de Alexandria», O Estudo da História, $n^{\circ}$ 6, Lisboa: Associação de Professores de História, 57-76. (2007), Estudos de Egiptologia. Temáticas e problemáticas. Lisboa: Livros Horizonte. (2008), Poder e Iconografia no antigo Egipto. Lisboa: Livros Horizonte.

- I. SERAGELDIN (2002), Bibliotheca Alexandrina: The rebirth of the Library of Alexandria. Alexandria, Bibliotheca Alexandrina. (2007), A landmark building. Reflections on the Architecture of the Bibliotheca Alexandrina. Alexandria: Bibliotheca Alexandrina.

- M. SHOUCRI (1997), "Alexandrie hier et aujourd'hui», Le Monde Copte. Revue trimestrielle de la culture copte, $\mathrm{n}^{\circ \mathrm{s}} 27-28$, Paris: S. I. P. E., 63-69.

\section{Webgrafia}

- http://www.bibalex.gov.eg

- http://www.houseofptolemy.org/

- http://www.touregypt.net/library/revivald.htm

- http://www.jwillisphoto.com/flash.html

- http://www.suite101.com/article.cfm/4205/89065

- http://www.angelfire.com/al/alexandriano1/bibliot.html

- http://www.unesco.org/comnat/france/alexandrie.htm 